Supporting Information

\title{
Light-Stimulated Carbon Dot Hydrogel: Targeting and Clearing Infectious Bacteria In Vivo
}

Chang Heon Lee ${ }^{1 \dagger}$, Seuk Young Song ${ }^{2 \dagger}$, You Jung Chung ${ }^{1 \dagger}$, Eun Kyoung Choi ${ }^{2}$, Jinhyeong Jang ${ }^{1}$, Dai Heon Lee ${ }^{3}$, Hae Dong Kim², Dong-Uk Kim ${ }^{2 *}$, and Chan Beum Park ${ }^{{ }^{*}}$

${ }^{1}$ Department of Materials Science and Engineering, Korea Advanced Institute of Science and Technology (KAIST), Daejeon 34141, Republic of Korea.

${ }^{2}$ Rare Disease Research Center, Division of Biomedical Research, Korea Research Institute of Bioscience and Biotechnology (KRIBB), Daejeon 34141, Republic of Korea.

${ }^{3}$ Department of Chemistry, Korea Advanced Institute of Science and Technology (KAIST), Daejeon 34141, Republic of Korea.

†These authors contributed equally to this work.

*E-mails: parkcb@kaist.ac.kr (C.B.P.); kimdongu@kribb.re.kr (D.-U.K)

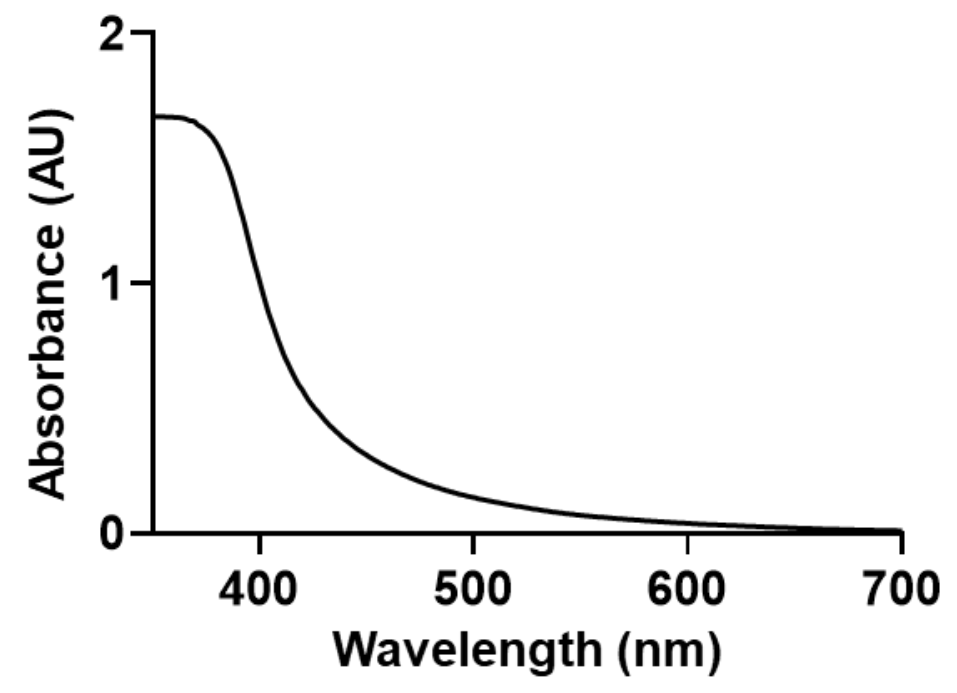

Figure S1. A representative absorption spectrum of CDs at $1 \mathrm{mg} \mathrm{mL}^{-1}$ concentration in water. CDs absorb broad ranges of light including UV and visible light. 

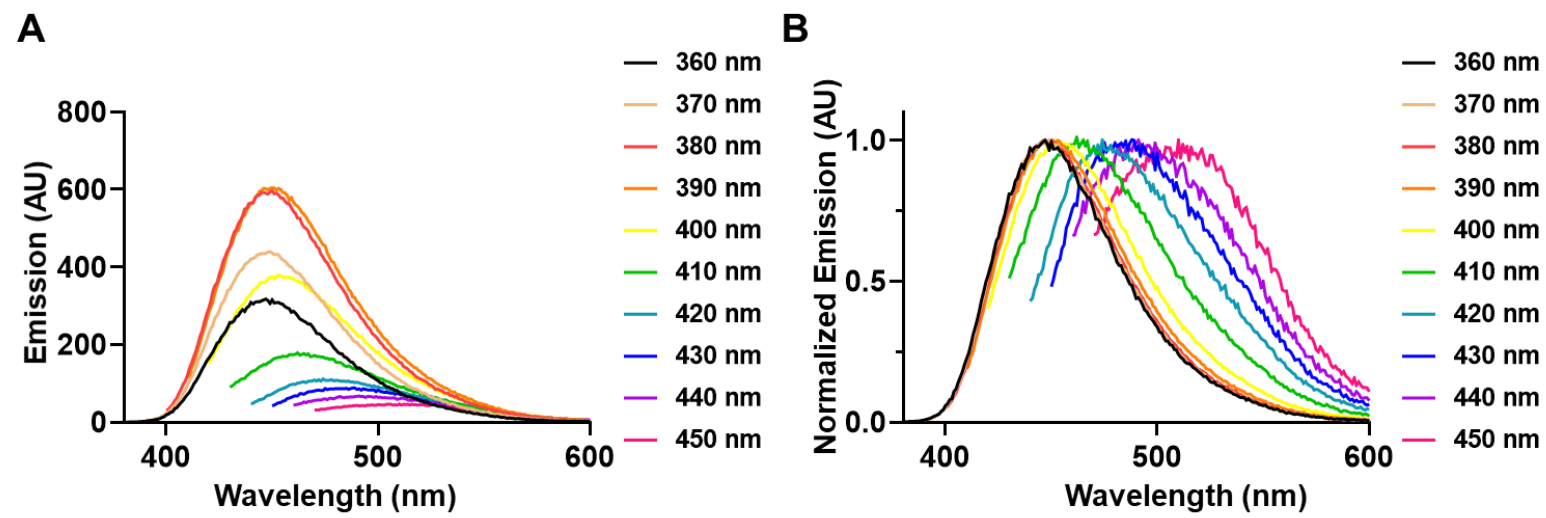

Figure S2. Photoluminescence characteristics of the CDs. (A) Raw emission spectra of the CDs at different excitation wavelengths. (B) Normalized PL spectra. Excitation wavelengths were varied from $360 \mathrm{~nm}$ to $450 \mathrm{~nm}$. 


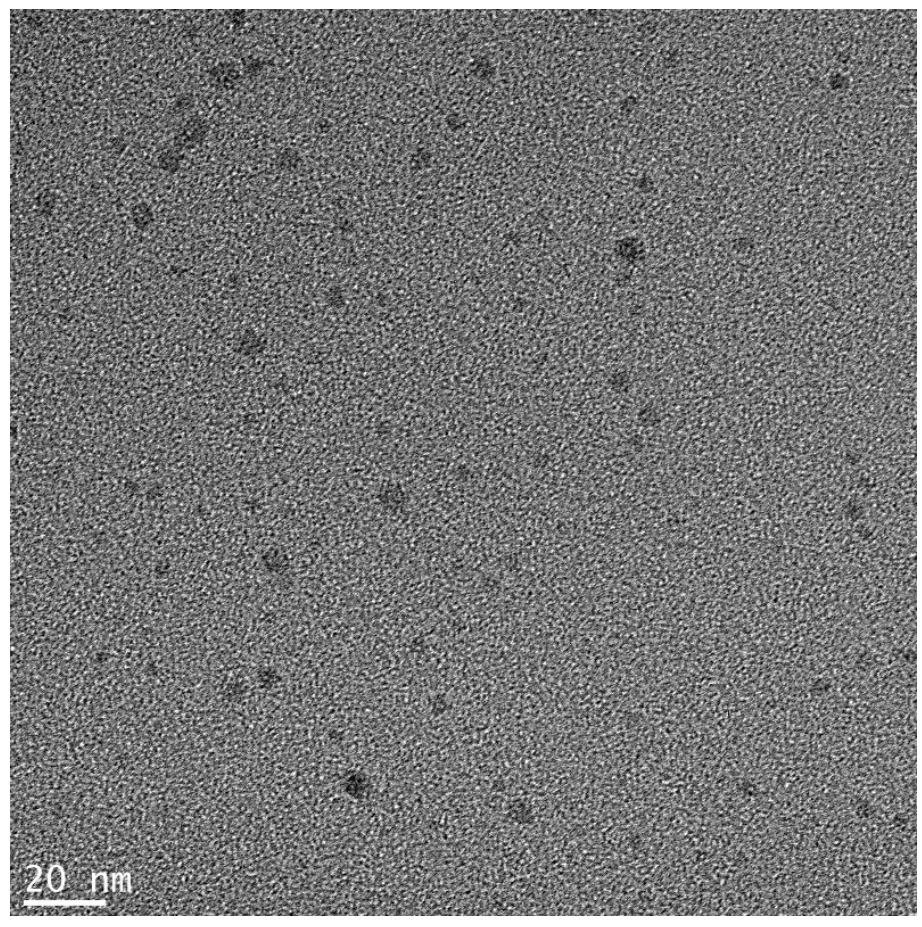

Figure S3. Exemplary TEM image of the CDs. 


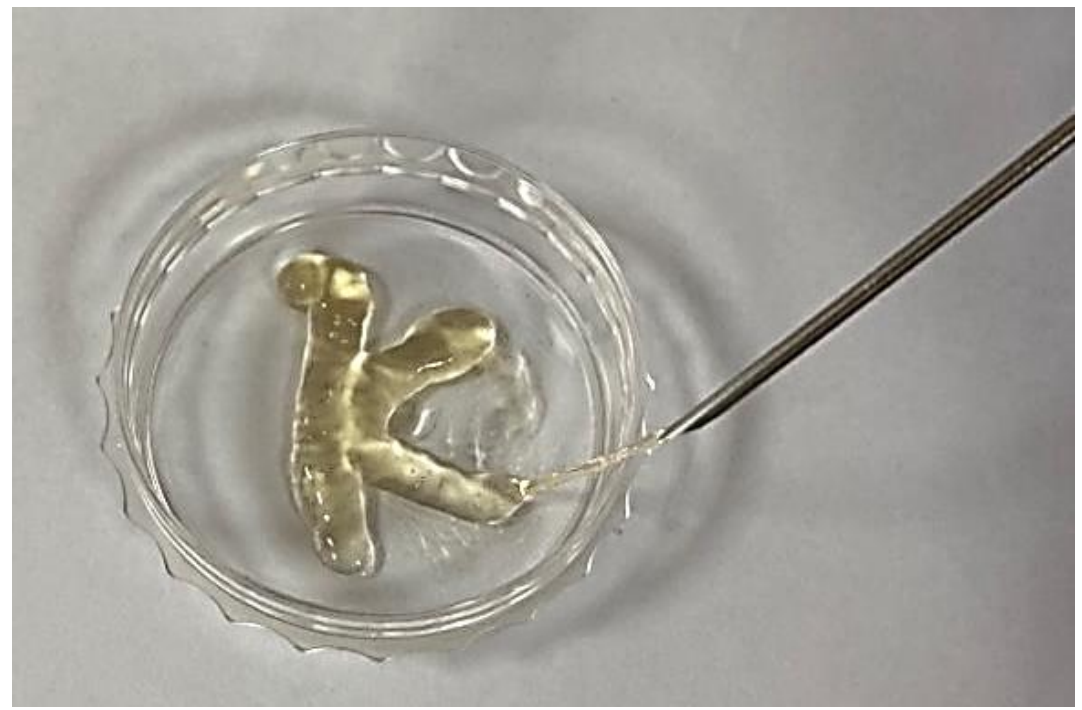

Figure S4. A representative photograph of the CDgel. The CDgel was soft enough to be transferred into a conventional syringe and injected through a needle to form a desired shape. 


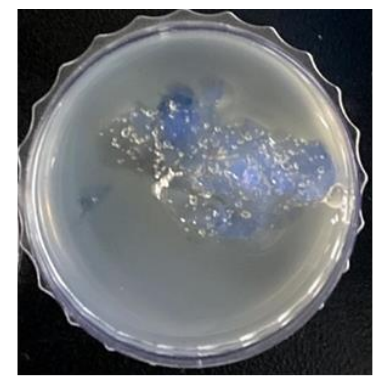

CDgel + E.coli under $U V$ at $t=0$

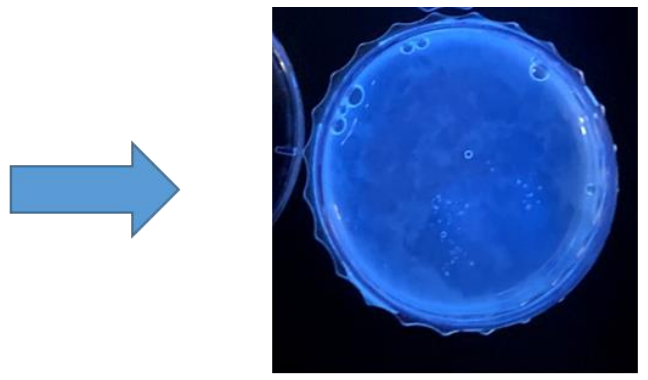

CDgel + E.coli under $U V$ after $2 \mathrm{~h}$ incubation

Figure S5. A representative image of CDgel in presence of E. Coli. The CDgel was incubated in E.coli containing broth culture for $2 \mathrm{~h}$. After $2 \mathrm{~h}$ incubation, the CDgel was fragmented and UV illumination indicated release of CDs. 


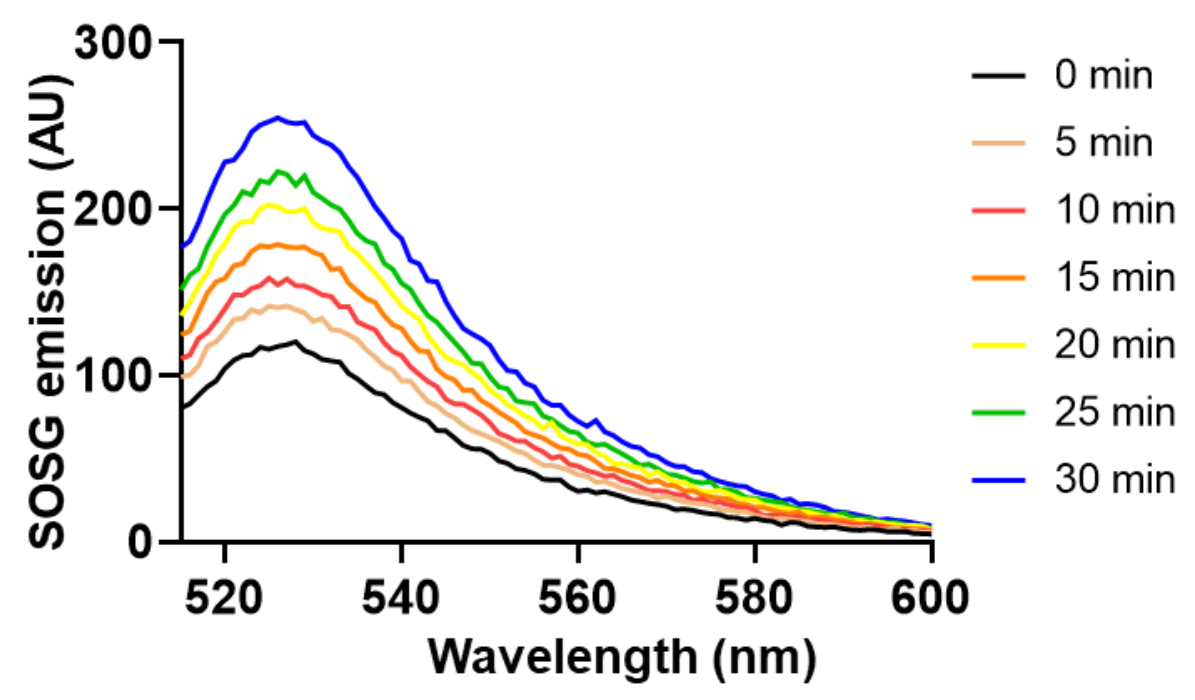

Figure S6. SOSG assay with free CDs. A SOSG solution (10 $\mu \mathrm{L}, 0.5 \mathrm{mM}$ in $\mathrm{MeOH})$ was added to a CD solution in PBS buffer $(0.5 \mathrm{ml})$, and then illuminated by white LED $\left(\sim 5 \mathrm{~mW} \mathrm{~cm}{ }^{-2}\right)$. SOSG emission spectra were recorded in every $5 \mathrm{~min}$ (488 $\mathrm{nm}$ excitation). Note that the concentration of CD used for free CDs experiment was $100 \mu \mathrm{g} \mathrm{ml}^{-1}$, and the concentration of CD from degraded CDgel by $140 \mathrm{U} \mathrm{ml}^{-1}$ HAase was estimated $200 \mu \mathrm{g} \mathrm{ml}^{-1}$ to $250 \mu \mathrm{g} \mathrm{ml}^{-1}$. 


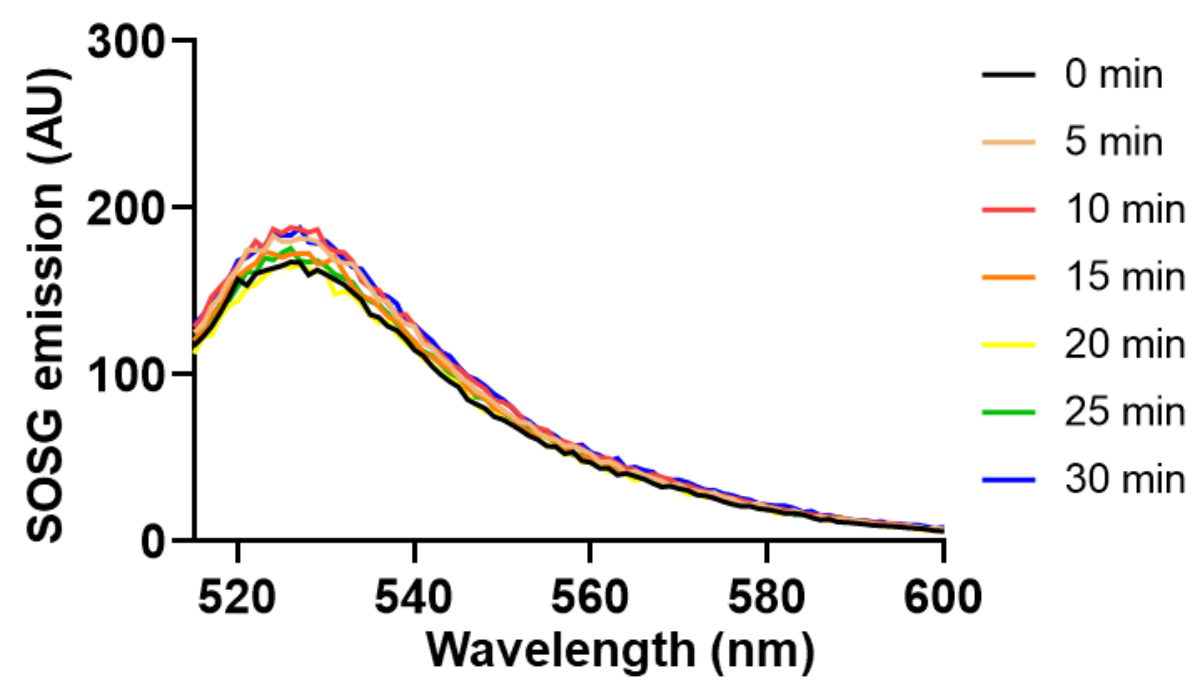

Figure S7. SOSG spectral changes with white LED illuminations without CDs. A SOSG solution $(10 \mu \mathrm{L}, 0.5 \mathrm{mM}$ in $\mathrm{MeOH})$ was added to a PBS buffer $(0.5 \mathrm{ml})$, and then illuminated by white LED $\left(\sim 5 \mathrm{~mW} \mathrm{~cm}^{-2}\right)$. Fluorescence spectra of SOSG at $525 \mathrm{~nm}$ were recorded in every 5 min (488 nm excitation). 


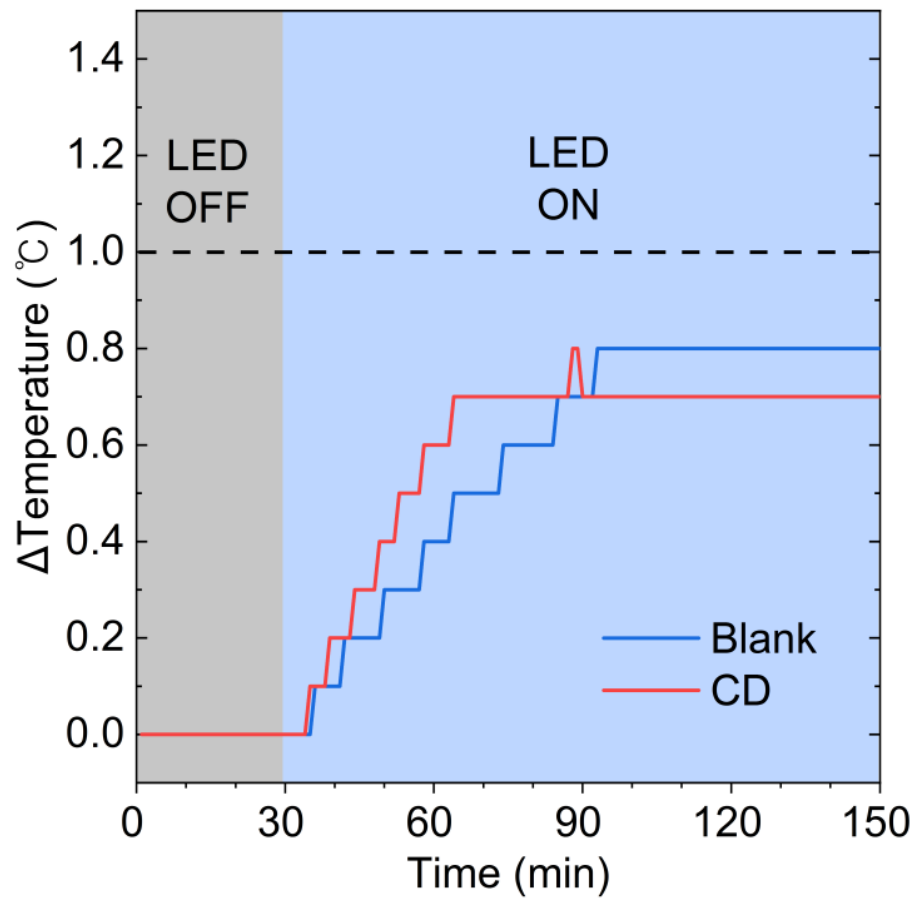

Figure S8. Temperature changes of CD solution upon illumination. 


\section{In vivo wound healing assay (E.coli)}

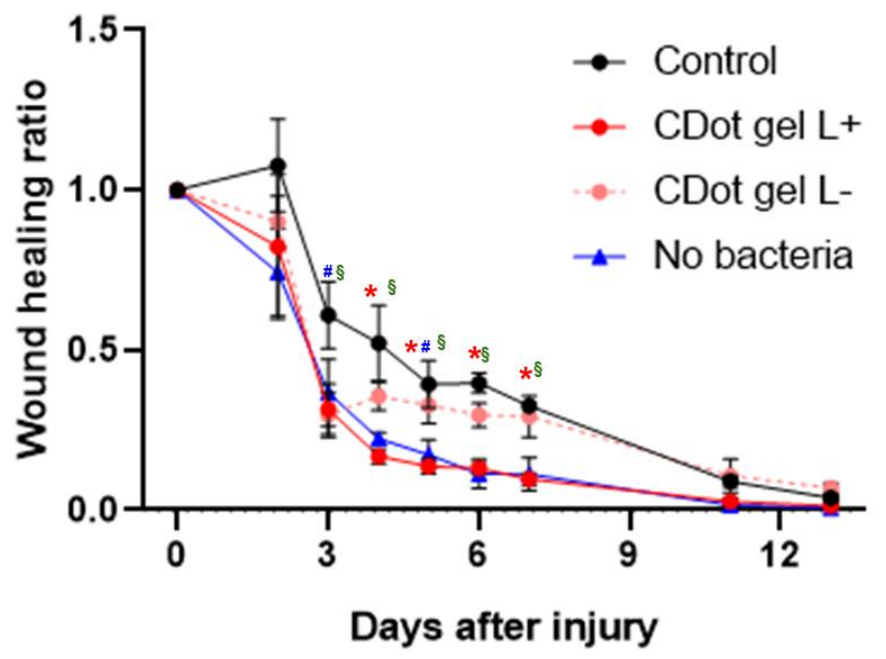

Figure S9. E.coli-infected wound healing assay with CDgel in the presence or absence of light. Infectious bacteria without any treatments were marked as Control and No bacteria group was wounds without bacterial infections. ${ }^{*} \mathrm{p}<0.05$; control group versus CDgel $+\mathrm{L}$ group, $\# \mathrm{p}<0.05$; control group versus CDgel group, $§ \mathrm{p}<0.05$; CDgel+L group versus CDgel group $(\mathrm{n}=8-12$ per group). 


\section{In vivo wound healing assay (S.aureus)}

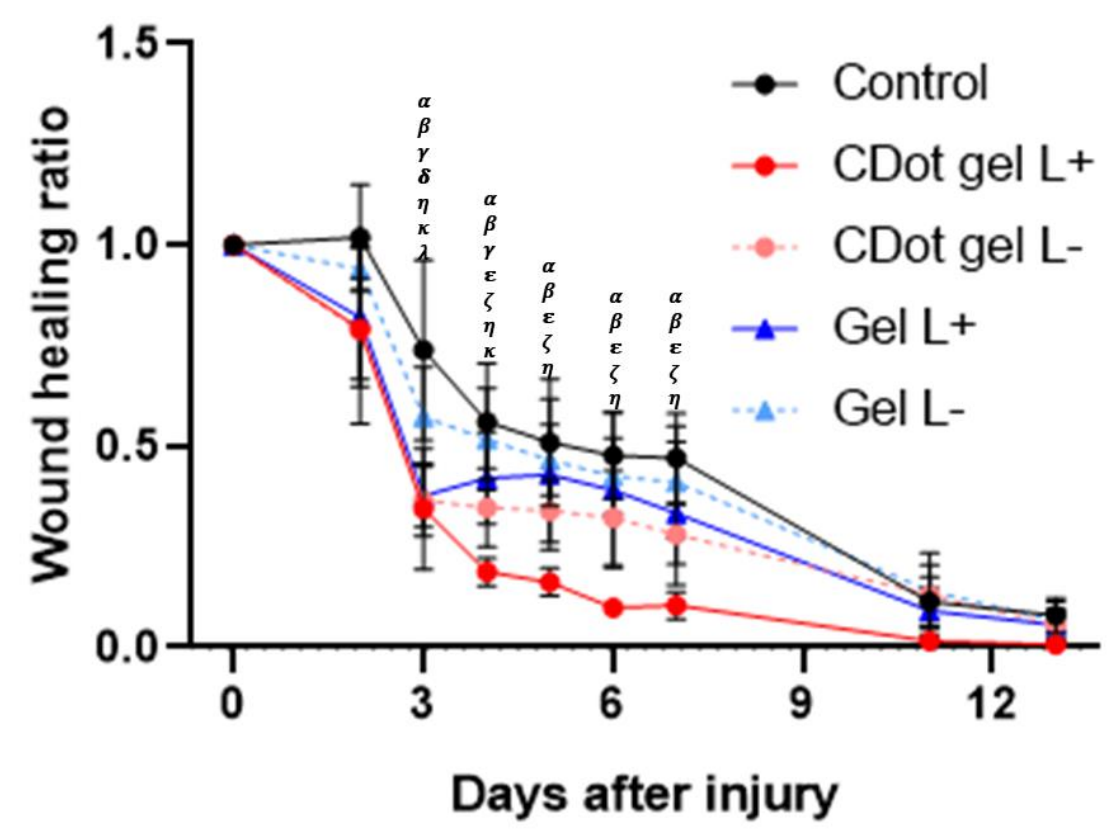

Figure S10. S. aureus-infected wound healing assay with $\mathrm{CDgel}$ in the presence or absence of light. Identical experiments were conducted by using gel without CDs (HAgel). Infectious bacteria without any treatments were marked as Control. ${ }^{\alpha} \mathrm{p}<0.05$; control group versus CDgel $+\mathrm{L}$ group, ${ }^{\beta} \mathrm{p}<0.05$; control group versus CDgel group, ${ }^{\gamma} \mathrm{p}<0.05$; control group versus HAgel+L group, $\delta_{\mathrm{p}}<0.05$; control group versus HAgel group, ${ }^{\varepsilon} \mathrm{p}<0.05$; CDgel $+\mathrm{L}$ group versus CDgel group, ${ }_{\mathrm{p}} \mathrm{p}<0.05$; CDgel+L group versus HAgel $+\mathrm{L}$ group, ${ }^{\eta} \mathrm{p}<0.05 ; \mathrm{CDgel}+\mathrm{L}$ group versus HAgel group, ${ }^{\vartheta} \mathrm{p}<0.05$; CDgel group versus HAgel $+\mathrm{L}$ group, ${ }^{\kappa} \mathrm{p}<0.05$; CDgel group versus HAgel group, ${ }^{\lambda} \mathrm{p}<0.05 ; \mathrm{HAgel}+\mathrm{L}$ group versus HAgel group ( $\mathrm{n}=8-12$ per group). 